\title{
Comprehensive analysis and optimal design of top-emitting organic light-emitting devices
}

\author{
Xue-Wen Chen \\ Department of Electrical and Electronic Engineering, University of Hong Kong, Pokfulam Road, \\ Hong Kong, China; Centre for Optical and Electromagnetic Research, Zhejiang University, Hangzhou \\ 310058, China; Joint Research Centre of Photonics of the Royal Institute of Technology, Sweden; \\ and Zhejiang University, Zhijingang campus, Hangzhou 310058, China \\ Wallace C. H. Choy ${ }^{\text {a) }}$ \\ Department of Electrical and Electronic Engineering, University of Hong Kong, Pokfulam Road, \\ Hong Kong, China \\ Sailing $\mathrm{He}$ \\ Centre for Optical and Electromagnetic Research, Zhejiang University, Hangzhou 310058, China; \\ Joint Research Centre of Photonics of the Royal Institute of Technology, Sweden; and Zhejiang University, \\ Zhijingang campus, Hangzhou 310058, China \\ P. C. Chui \\ Department of Electrical and Electronic Engineering, University of Hong Kong, Pokfulam Road, \\ Hong Kong, China
}

(Received 13 February 2007; accepted 6 April 2007; published online 12 June 2007)

\begin{abstract}
We present an accurate analysis of light emission in top-emitting organic light-emitting devices (TOLEDs) by explicitly considering the Purcell effect. TOLEDs are optimized separately for maximum zero-degree luminance, maximum electroluminescence (EL) efficiency, and wide viewing angle with high EL efficiency. For fluorescent material with an internal quantum efficiency $\left(\eta_{\text {int }}^{0}\right)$ of 0.25 , the maximum zero-degree luminance and EL efficiency can be achieved by locating the emitters around the first antinode of the microcavity while for phosphorescent material with $\eta_{\text {int }}^{0}=1.0$, the maximum zero-degree luminance and EL efficiency are around the second antinode. Through relaxing the efficiency by $10 \%-20 \%$, the angular intensity distribution can be even better than the Lambertian distribution; meanwhile, the color shows only a small variation over an angle range of $150^{\circ}$. Our results, which are in good agreement with experiments, show that the Purcell effect on TOLED performances is significant and should be carefully examined in studying TOLEDs. @ 2007 American Institute of Physics. [DOI: 10.1063/1.2739220]
\end{abstract}

\section{INTRODUCTION}

Top-emitting organic light-emitting devices (TOLEDs), which allow the fabrication of OLEDs on silicon wafers with active-matrix backplanes, have been the subject of intensive research recently. ${ }^{1-8} \mathrm{~A}$ typical TOLED, as shown on the lefthand side of Fig. 1, consists of a bottom reflective metal contact as the anode, organic layers, a top semitransparent metal contact as the cathode, and a high-index capping layer for output enhancement. The strong microcavity formed between the two metal electrodes not only influences the photon outcoupling efficiency but also significantly modifies the molecule's spontaneous emission rate. ${ }^{9,10}$ The latter effect is the so-called Purcell effect, ${ }^{11}$ which can alter the lifetime of the excitons and the ratio of radiative and nonradiative decay rate, resulting in a change of the internal quantum efficiency (IQE) ${ }^{12,13}$ Since the external quantum efficiency is the product of IQE and the photo outcoupling efficiency, the Purcell effect, particularly in top-emitting devices, plays an important role in the device performance. However, in the analysis of TOLED, the Purcell effect has not been treated properly.

\footnotetext{
${ }^{\text {a) }}$ Author to whom correspondence should be addressed; telephone: (852) 2857-8485; FAX: (852) 2559-8738; electronic mail: chchoy@eee.hku.hk
}

For example, a widely used approach on the analysis of electroluminescence (EL) efficiency of TOLED considers only the photo outcoupling efficiency, ${ }^{5}$ which usually results in underestimating the EL efficiency. Some other theoretical analysis $^{7,12}$ overlooks the modification of the exciton lifetime in the microcavity, overestimating the efficiency enhancement. This error can be noticeably large when the emitting
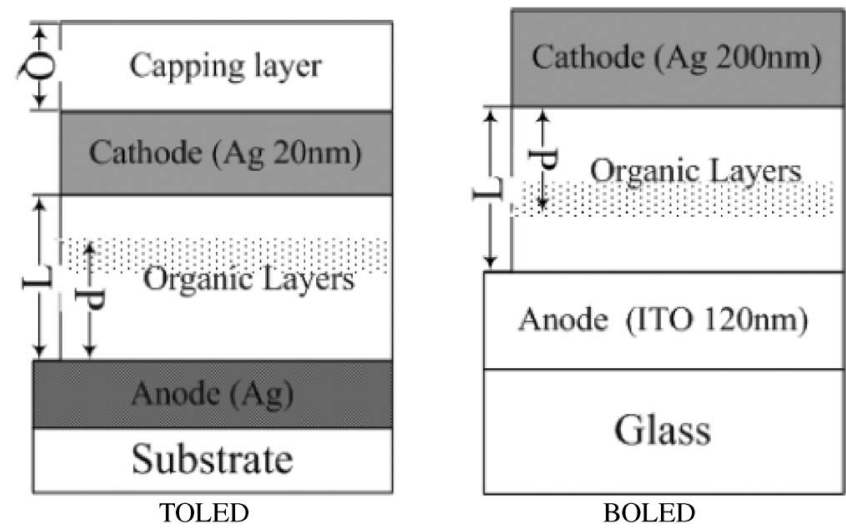

FIG. 1. Schematic diagrams of a TOLED and BOLED, where $L, P$, and $Q$ are the three design parameters. 
material has an IQE of 1.0, such as some phosphorescent materials. The Purcell effect is not only important for the accurate assessment of TOLED performance but also useful for boosting the device efficiency through a proper design.

Another important issue of the microcavity-based OLEDs is the relatively strong directionality of the emission. ${ }^{12,14}$ Highly angle-dependent color or intensity of the emission in most cases is a problem for flat panel display applications. Optimization of TOLEDs for simultaneously achieving a wide viewing angle and a high EL efficiency is challenging ${ }^{6}$ but desirable for display applications.

In this article we present an accurate and efficient analysis of TOLEDs by explicitly taking the Purcell effect into account, and then optimize the devices separately for (i) maximum zero-degree luminance, (ii) maximum EL efficiency, and (iii) wide viewing angle with high EL efficiency. With the consideration of the Purcell effect, we find the fluorescent organic emitter near the first antinode in the microcavity is more efficient than that near the second antinode, which is contradictory to the prediction made in Ref. 15 (due to the negligence of the Purcell effect). Moreover, by relaxing the EL efficiency by $10 \%$ to $20 \%$, the angular emission characteristics of the TOLEDs can be considerably improved so that the angular intensity distribution is even better than the Lambertian distribution while the angular color variation is very small. The article is organized as follows. In Sec. II, theoretical analysis is described. The methodologies for device optimization are addressed in Sec. III. In Sec. IV, results are presented and discussed in detail. Finally, a conclusion is given in Sec. V.

\section{THEORETICAL ANALYSIS}

The theoretical analysis of an OLED is based on a classical approach in which the recombination zone of the OLED is assumed to be $10 \mathrm{~nm}$ thick and contains an ensemble of mutually incoherent and uniformly distributed electric dipoles running at a fixed current with random orientations in space. ${ }^{9,16}$ To analyze the radiation properties of the incoherent dipoles with random orientations, one only needs to consider two kinds of electric dipoles, namely, the vertical electric dipole and horizontal electric dipole. The total radiation power of the electric dipoles and the power extracted to the far-field region should be calculated, since the former term can be used to characterize the Purcell effect while the ratio of the latter to the former term gives the outcoupling efficiency. The radiation field of an electric dipole in an infinite medium can be decomposed into plane waves with TE and TM polarization. In the multilayered structure, reflection and transmission of each plane wave occur at interfaces, and the resulting electric field in the emitting layer can be represented by a Fourier integral of the upward and downward plane waves. The total radiation power $F$, the extracted power $U$, and angular power density $S$, normalized by the radiation power of the dipole in the infinite medium, can be expressed as ${ }^{16,17}$

$$
F(\lambda)=\int_{0}^{\infty} \mathfrak{R}\left(f\left(\lambda, k_{\rho}\right)\right) d k_{\rho}
$$

$$
\begin{aligned}
& U(\lambda)=\int_{0}^{\infty} \Re\left(u\left(\lambda, k_{\rho}\right)\right) d k_{\rho}, \\
& S(\alpha, \lambda)=k^{2} \cos \alpha u(k \sin \alpha) / \pi,
\end{aligned}
$$

where $\Re(\cdot)$ stands for the real part of $(\cdot), \alpha$ is the viewing angle. and $k$ is the wavenumber in the outermost medium; $\lambda$ is the wavelength and $k_{\rho}$ is the radial component of the wave vector in cylindrical coordinates; $f$ and $u$ are the averaged results of the electric dipole radiating with random orientations in space. Their detailed expressions can be found in Ref. 17. Direct evaluation of the integral in Eq. (1) tends to fail since the integrand has pole singularities, which correspond physically to the modes guided in the multilayered structure. Here, we employ a technique proposed in Ref. 17 to perform the integration along an alternative path in the complex plane of $k_{\rho}$. By using a Gaussian quadrature, ${ }^{18}$ the integrals of Eq. (1) and (2) can be evaluated very efficiently.

In the microcavity structure, the radiative decay rate of excitons $\Gamma_{r}^{m}$ is modified to be ${ }^{16}$

$$
\Gamma_{r}^{m}=F \cdot \Gamma_{r}^{0},
$$

where $\Gamma_{r}^{0}$ is the radiative decay rate in the infinite medium. Equation (4) is a consequence of Fermi's Golden Rule for the radiative decay rate since the total radiated power also describes the local density of states available for an emitter. ${ }^{19}$ With a typical assumption that the nonradiative decay rate $\Gamma_{\text {nr }}$ is a constant, the IQE $\eta_{\text {int }}^{\text {cav }}$ and exciton lifetime $\tau_{\text {cav }}$ in the microcavity become

$$
\begin{aligned}
& \eta_{\text {int }}^{\mathrm{cav}}=\Gamma_{r}^{m} /\left(\Gamma_{r}^{m}+\Gamma_{\mathrm{nr}}\right)=F /\left[(F-1) \eta_{\mathrm{int}}^{0}+1\right] \cdot \eta_{\mathrm{int}}^{0}, \\
& \tau_{\mathrm{cav}} / \tau_{0}=\left(\Gamma_{r}^{0}+\Gamma_{\mathrm{nr}}\right) /\left(\Gamma_{r}^{m}+\Gamma_{\mathrm{nr}}\right)=1 /\left[(F-1) \eta_{\mathrm{int}}^{0}+1\right],
\end{aligned}
$$

where $\tau_{0}$ and $\eta_{\text {int }}^{0}$ are the exciton lifetime and IQE of the bulk emitting material, respectively. The photon outcoupling efficiency $\eta_{\mathrm{cp}}(\lambda)$ is defined as

$$
\eta_{\mathrm{cp}}(\lambda)=U(\lambda) / F(\lambda) \text {. }
$$

Due to the change of IQE in the microcavity [see Eq. (5)], the photon outcoupling efficiency should be modified as

$$
\eta_{\mathrm{cp}}^{m}(\lambda)=U(\lambda) /\left(1+(F(\lambda)-1) \eta_{\mathrm{int}}^{0}\right),
$$

where $\eta_{\mathrm{cp}}^{m}(\lambda)$ is the modified photon outcoupling efficiency taking the Purcell effect into account. Accordingly the angular power density will also be modified as

$$
s(\alpha, \lambda)=S(\alpha, \lambda) /\left(1+(F-1) \eta_{\text {int }}^{0}\right) .
$$

The above analysis on the dipole radiation is only for one wavelength at a fixed position. It should be noted that the emission characteristics of an OLED are obtained by averaging the dipole radiation over the recombination zone and wavelength of interest. Thus we define an integrated outcoupling efficiency $\eta$ and angular intensity distribution $I(\alpha)$ to characterize a TOLED, 


$$
\begin{gathered}
\eta=\int_{\lambda_{1}}^{\lambda_{2}} \eta_{c p}^{m}(\lambda) s_{0}(\lambda) d \lambda / \int_{\lambda_{1}}^{\lambda_{2}} s_{0}(\lambda) d \lambda, \\
I(\alpha)=\int_{\lambda_{1}}^{\lambda_{2}} s(\alpha, \lambda) d \lambda / \int_{\lambda_{1}}^{\lambda_{2}} s_{0}(\lambda) d \lambda,
\end{gathered}
$$

where $\left(\lambda_{1}, \lambda_{2}\right)$ is the considered wavelength range and $s_{0}(\lambda)$ is the intrinsic emission spectrum of the emitting material. The EL efficiency of the TOLED $\eta_{\text {ext }}$ is now given as

$$
\eta_{\text {ext }}=\eta_{\text {int }}^{0} \cdot \eta
$$

Thus we need to maximize $\eta$ for optimizing EL efficiency.

\section{OPTIMIZATION METHODOLOGY}

In this section, we will present the methodologies for optimizing TOLEDs to achieve separately (i) the maximum zero-degree luminance, (ii) the maximum EL efficiency, and (iii) wide viewing angle with high EL efficiency. The numerical details will also be given.

As shown on the left-hand side of Fig. 1, there are three design parameters for a TOLED, namely, the total thickness of organic layers $L$, position of the recombination zone $P$, and thickness of the capping layer $Q$. In a real device, $L, P$, and $Q$ can be controlled by tailoring the thickness of the hole and electron transport layers and other layers used in the device. By employing the efficient model in Sec. II, one can simply optimize the three parameters by a global scanning. The scanning can be done in a few minutes on a personal computer. Consequently, it is straightforward to find a TOLED structure to achieve the maximum zero-degree luminance or the maximum EL efficiency according to Eqs. (10) and (11). However, further physical considerations and treatments are required to optimize TOLED for achieving a wide viewing angle with high EL efficiency since the color shift, intensity variation over the angles, and EL efficiency should be considered simultaneously. Here we carry out the optimization of a device structure of which $\eta$ and color shift over an angle range from $0^{\circ}$ to $75^{\circ}$ (measured from the normal direction of the emitting surface) are better than the preset levels, and in the mean time the angular intensity variation is minimum. Mathematically, the optimization for a wide viewing angle with high EL efficiency is achieved when

$$
[1-\min [I(\alpha)] / \max [I(\alpha)]] \rightarrow \min
$$

under the conditions of

$$
\begin{aligned}
& \eta \geq \gamma \cdot \eta_{\max }, \\
& \max [X(\alpha)]-\min [X(\alpha)] \leq \mathrm{s},
\end{aligned}
$$

where $\eta_{\max }$ is the maximum integrated outcoupling efficiency obtained by optimizing TOLED for maximum EL efficiency using Eq. $(10) ; X(\alpha)$ is the $x$ coordinate in the CIE (Commission Internationale de l'Eclairage) chromaticity diagram of the emission at the viewing angle $\alpha ; \gamma$ and $\zeta$ are the preset levels of the efficiency and tolerance of color shift, respectively. Here $\min [\cdot]$ and $\max [\cdot]$ stand for the minimum and maximum of $[\cdot]$ within the angle range from $0^{\circ}$ to $75^{\circ}$, respectively. The color shift over the viewing angles should be inappreciable, and thus here we set $\zeta$ to be 0.03 . Then we optimize the TOLEDs to obtain a minimum angular intensity variation at three different levels of EL efficiencies, i.e., $\gamma$ $=0.8,0.85$, and 0.9 .

In the calculations, the refractive indices of the organic materials and the capping layer are assumed to be 1.77 and 2.2 over the wavelength of interest, respectively. The metallic electrode used here is silver $(\mathrm{Ag})$ and its complex permittivity is taken from Ref. 20. As shown on the right-hand OLED structure of Fig. 1, a bottom emitting OLED is also studied for comparison. The ITO thickness is measured in house while $L$ and $P$ are the two free design parameters. The refractive index of the ITO is taken from Ref. 21. The intrinsic emission spectrum $s_{0}(\lambda)$ is assumed to be a Gaussian shape with a peak at $530 \mathrm{~nm}$ and a full-width at half maximum of $80 \mathrm{~nm}$. For the IQE of bulk emitting material, $\eta_{\text {int }}^{0}$ $=0.25$ and $\eta_{\text {int }}^{0}=1.0$ are used for the fluorescent and phosphorescent materials, respectively.

\section{RESULTS AND DISCUSSIONS}

In this section, interesting results for the optimization of TOLEDs will be given and discussed in detail. The devices separately optimized for maximum zero-degree luminance and EL efficiency will be discussed in Sec. IV A. We will also show that our numerical results are in good agreement with reported experiments. In Sec. IV B, the optimized results for a wide viewing angle with high EL efficiency will be delivered.

\section{A. The maximum zero-degree luminance and $E L$ efficiency}

The lines with filled symbols in Fig. 2(a) show the dependences of the maximum of zero-degree luminance [defined in Eq. (11) at zero degree] $I_{o}$ and the maximum of integrated outcoupling efficiency [defined in Eq. (10)] $\eta$ on the total thickness of the organic layers. It is important to note that the values of the $I_{o}$ and $\eta$ shown in Fig. 2(a) for every $L$ (i.e., $x$ axis) are locally maximized by optimizing the other two device parameters $P$ and $Q$. Due to the Fabry-Pérot effect, ${ }^{22} I_{o}$ and $\eta$ show periodic oscillation features. The devices correspond to the highest $I_{o}$ and $\eta$ are denoted by $H_{-} I_{o}$ and $H_{-} \eta$, respectively. Their device parameters and the integrated outcoupling efficiency are listed in column 2 and 3 of Table I. From Fig. 2(a), one can find that the trend of the $I_{o}$ and $\eta$ shows similar oscillating features. Therefore, to avoid duplicating discussion, we will focus on analyzing the results of $\eta$ in the following discussions.

The EL efficiency of an OLED with defined organic materials is proportional to $\eta$ as shown in Eq. (12). Thus $\eta$ will be discussed hereafter for the EL efficiency. The device corresponding to the second peak of $\eta$ in Fig. 2(a) is denoted by $B$, and its parameters are listed in columns 4 of Table I. From Fig. 2(a), one sees that with the consideration of the Purcell effect the emitter near the first antinode (device $H_{-} \eta$ ) is much more efficient than the emitter near the second antinode (device $B)$ for the case of fluorescent material $\left(\eta_{\text {int }}^{0}\right.$ $=0.25$ ), which is inconsistent with the predictions of the highest at the second antinode in Ref. 15 due to an oversight 

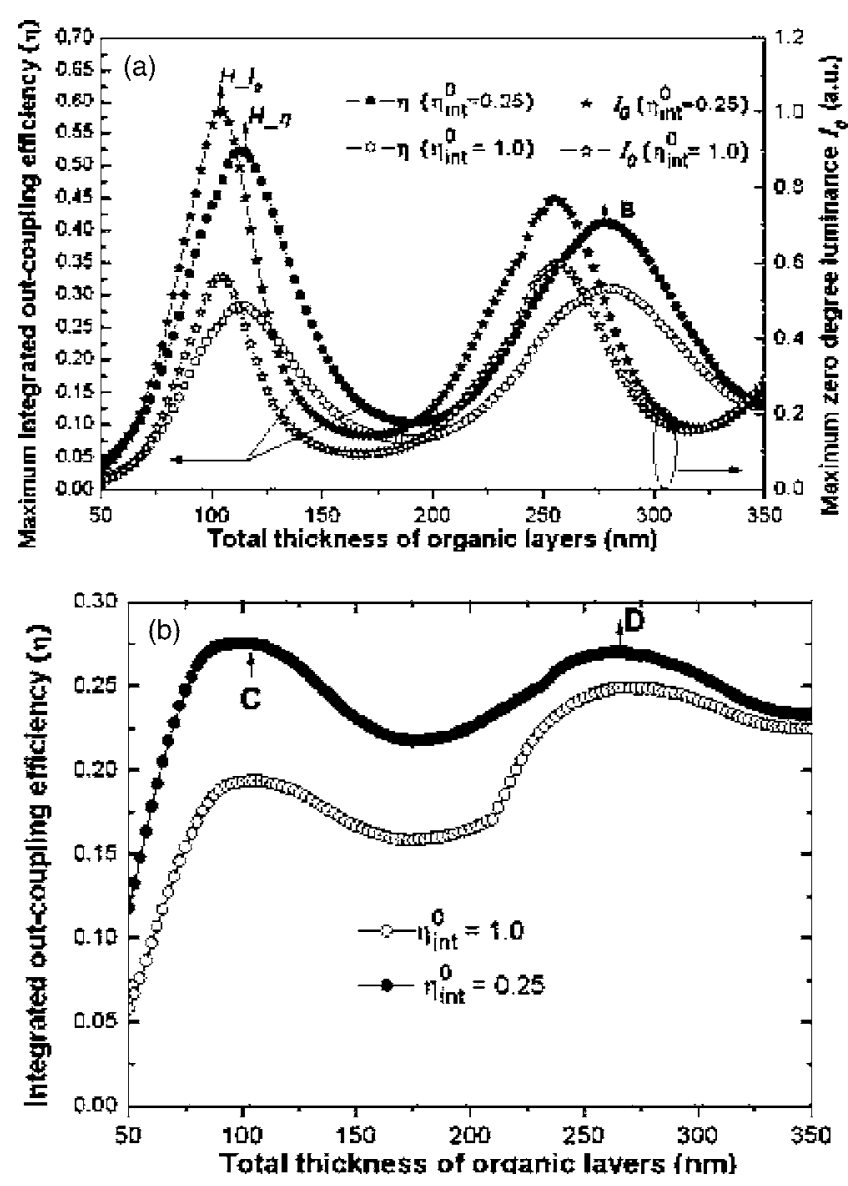

FIG. 2. (a) The maximum integrated outcoupling efficiency $\eta$ and maximum zero-degree luminance $I_{o}$ of TOLED with the variance of the total thickness of the organic layers. The highest points of the $\eta$ on the lines are denoted as $H_{-} \eta$ and $B$. (b) The maximum integrated outcoupling efficiency $\eta$ of BOLED with variance of the total thickness of the organic layers. The peak points of the $\eta$ on the lines are denoted as $C$ and $D$.

of the change in the IQE in Ref. 15. This can be confirmed by the plots [with open symbols in Fig. 2(a)] of the dependences of $I_{o}$ and $\eta$ on $L$ without considering the IQE change, i.e., setting $\eta_{\text {int }}^{0}=1.0$. In this case, the emitter around the second antinode is more efficient than the emitter near the first antinode as predicted in Ref. 15. But for fluorescent emitter of $\eta_{\text {int }}^{0}=0.25$, the IQE change must be considered and the highest $\eta$ should be the TOLED with emitters near the first antinode. For comparison, we also optimize a BOLED shown on the right-hand OLED structure of Fig. 1 for maximum EL efficiency. Figure 2(b) plots the dependences of the maximum integrated $\eta$ on the total thickness of organic layers. One sees that for $\eta_{\text {int }}^{0}=0.25$ device $C$ is still more efficient than device $D$ but the outcoupling efficiency difference is much smaller than the difference between device $H_{-} \eta$ and $B$. This is due to a weaker Purcell effect in BOLED. The parameters and integrated outcoupling efficiencies of devices $C$ and $D$ are also listed in Table I. As a consequence, our results show that for fluorescent material of $\eta_{\text {int }}^{0}=0.25$, the highest $I_{o}$ and $\eta$ can be obtained by locating the emitters around their first antinode, while for phosphorescent material of $\eta_{\text {int }}^{0}=1.0$, the highest $I_{o}$ and $\eta$ are around the second antinode. Thus, a careful study of the Purcell effect is critical for separately optimizing TOLEDs for maximum zero-degree luminance and maximum EL efficiency.
TABLE I. Design parameters and $\eta$ of the optimized devices.

\begin{tabular}{ccccccccc}
\hline \hline & $H_{-} I_{o}$ & $H_{-} \eta$ & $B$ & $C$ & $D$ & $\gamma=0.8$ & $\gamma=0.85$ & $\gamma=0.90$ \\
\hline$L(\mathrm{~nm})$ & 105 & 113 & 277 & 105 & 268 & 118 & 118 & 113 \\
$P(\mathrm{~nm})$ & 60 & 65 & 220 & 75 & 220 & 68 & 68 & 68 \\
$Q(\mathrm{~nm})$ & 68 & 68 & 60 & $/$ & $/$ & 160 & 168 & 168 \\
$\eta$ & 0.44 & 0.52 & 0.41 & 0.28 & 0.27 & 0.43 & 0.45 & 0.48 \\
\hline \hline
\end{tabular}

To illustrate the Purcell effect more clearly, we calculate the change of IQE and exciton lifetime for all four devices and list the results in Table II. The results have been averaged over the wavelength of interest. We see that the increment of IQE for fluorescent material in TOLED is considerably larger than in BOLED. Device $H_{-} \eta$ has an increment of $84 \%$ in IQE for fluorescent material. However, even in the BOLED (like device $C$ ), the Purcell effect cannot be neglected for analyzing and optimizing device structures. In fact, it has been reported that the IQE is increased to $35 \%-$ $45 \%$ for the conjugated polymer-based emitters in a BOLED, ${ }^{23}$ which agrees with our calculation. The effect of the IQE increment was also reported in InGaN-based emitters. ${ }^{13}$ As shown in Table II, the change of lifetime is also more prominent in TOLED than in BOLED. The results show that the conventional assumption of $\tau_{\text {cav }} / \tau_{\text {con }} \cong 1$ for the analysis of a TOLED ${ }^{7,12}$ will overestimate the EL efficiency. Particularly for the phosphorescent TOLED with $\eta_{\text {int }}^{0}=1.0$, the overestimation is as large as $38 \%$ (see the lifetime of device $A$ for $\eta_{\text {int }}^{0}=1.0$ ), but for fluorescent material, the overestimation is smaller. For example, $\tau_{\text {cav }} / \tau_{\text {con }}=0.816$ for device $H_{-} \eta$ and $C$. Another confirmation of our simulated result is the experiment reported in Ref. 7, where a nearly $20 \%$ reduction of the lifetime of the fluorescent emitter was observed. Consequently, apart from analyzing and optimizing TOLEDs for the maximum zero-degree luminance and EL efficiency, we also clearly show that the Purcell effect is important for accurate assessment and optimal design of not only TOLEDs but even weak cavity devices like conventional BOLEDs. Meanwhile, our simulated results are in good agreement with the experiments.

\section{B. Wide viewing angle with high EL efficiency}

For wide angle display applications, we optimize TOLED to achieve small angular intensity variation, color shift, and high EL efficiency according to Eqs. (13)-(15). The design parameters of the three optimized TOLEDs with efficiency levels of $\gamma=0.8,0.85$, and 0.9 are shown in the last three columns of Table I. For the five devices in Table I, namely $H_{-} I_{o}, H_{-} \eta, \gamma=0.8, \gamma=0.85$, and $\gamma=0.90$, we plot the angular intensity distributions and $x$-CIE coordinates in Figs. 3(a) and 3(b), respectively. The standard Lambertian distribution is shown as the solid line in Fig. 3(a) for reference. The results show that the emission of devices $H_{-} I_{o}$ and $H_{-} \eta$ is highly angle dependent. It has been recently reported $^{6}$ that it is difficult to design a TOLED with the angular intensity distribution close to the Lambertian distribution. Our results show that by simultaneously optimizing $L, P$, and $Q$ through Eq. (13), the angular viewing characteristics can be considerably improved by relaxing 10\%-20\% 
TABLE II. Internal quantum efficiencies (IQE) and lifetime of excitons in devices $H_{-} \eta, B, C$, and $D$.

\begin{tabular}{|c|c|c|c|c|c|c|c|c|}
\hline & \multicolumn{2}{|c|}{$H_{-} \eta$} & \multicolumn{2}{|c|}{$B$} & \multicolumn{2}{|c|}{ C } & \multicolumn{2}{|c|}{$D$} \\
\hline & IQE & $\tau_{\text {cav }} / \tau_{0} 0$ & IQE & $\tau_{\mathrm{cav}} / \tau_{0}$ & IQE & $\tau_{\text {con }} / \tau_{0}$ & IQE & $\tau_{\text {con }} / \tau_{0}$ \\
\hline$\eta_{\mathrm{int}}^{0}=0.25$ & 0.46 & 0.71 & 0.33 & 0.89 & 0.36 & 0.87 & 0.27 & 0.97 \\
\hline$\eta_{\mathrm{int}}^{0}=1.0$ & 1.0 & 0.38 & 1.0 & 0.67 & 1.0 & 0.61 & 1.0 & 0.90 \\
\hline
\end{tabular}

of the efficiency. The angular intensity distributions of the three devices optimized for a wide viewing angle are all better than the Lambertian distribution, particularly for device $\gamma=0.8$. The emission spectra of device $H_{-} \eta$ and device $\gamma=0.85$ at various viewing angles are shown in Figs. 4(a) and 4(b), respectively. Compared with Fig. 4(a), Fig. 4(b) shows a much smaller spectral shift over the viewing angles. From the results in Table I, it is worth to note that for the fluorescent BOLED of device $C$, the EL efficiency is only $54 \%$ of that of device $H_{-} \eta$. As a result, the TOLED optimized for a wide viewing angle with $\gamma=0.8$ is still much more efficient than the BOLED. Thus we can obtain a TOLED that its angular intensity distribution can be even better than the Lambertian distribution; meanwhile, the color shows only a small variation over an angle range of $150^{\circ}$ and the EL efficiency can be still much more efficient than an optimized BOLED.

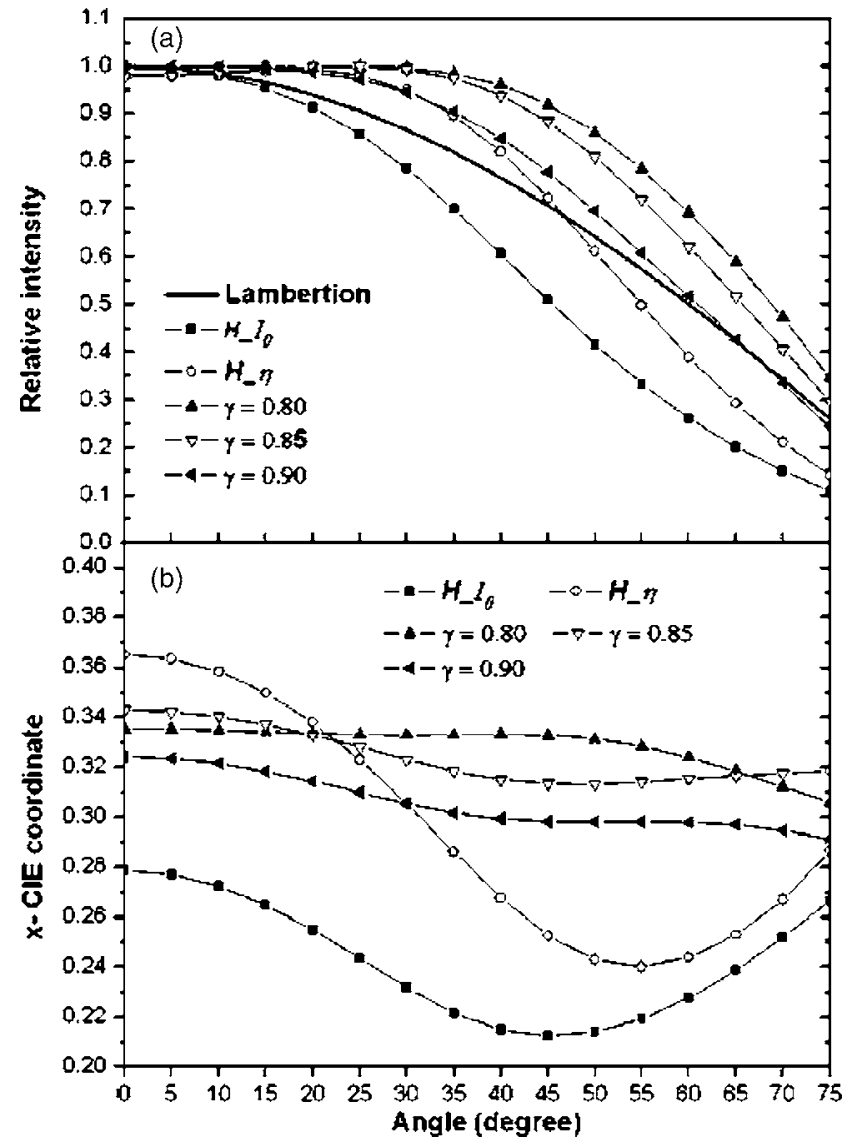

FIG. 3. Plots of (a) angular intensity distributions and (b) $x$-CIE coordinates of TOLEDs optimized for $I_{o}, \eta$ and wide viewing angles with $\gamma=0.80,0.85$, and 0.90 , respectively.

\section{CONCLUSIONS}

We have presented an accurate analysis of TOLEDs taking the Purcell effect into account. The separately optimized results for maximizing the zero-degree luminance, EL efficiency, and wide viewing angle with high EL efficiency are comprehensively discussed. For fluorescent material with an internal quantum efficiency $\left(\eta_{\text {int }}^{0}\right)$ of 0.25 , the highest zerodegree luminance and EL efficiency can be achieved by locating the emitters around the first antinode of the microcavity, while for phosphorescent material with $\eta_{\text {int }}^{0}=1.0$, the highest zero-degree luminance and EL efficiency are for the emitters around the second antinode. Through relaxing the EL efficiency by $10 \%-20 \%$, a wide viewing angle can be achieved in TOLED. We have shown that the Purcell effect is important for accurate assessment and optimal design of not only TOLEDs but also BOLEDs.

\section{ACKNOWLEDGMENTS}

We would like to acknowledge the support of a UDF grant, a strategic research grant in organic optoelectronics of the University of Hong Kong, and a grant (No. 14300.324.01) from the Research Grant Council of the Hong Kong Special Administrative Region, China. P.C. Chui

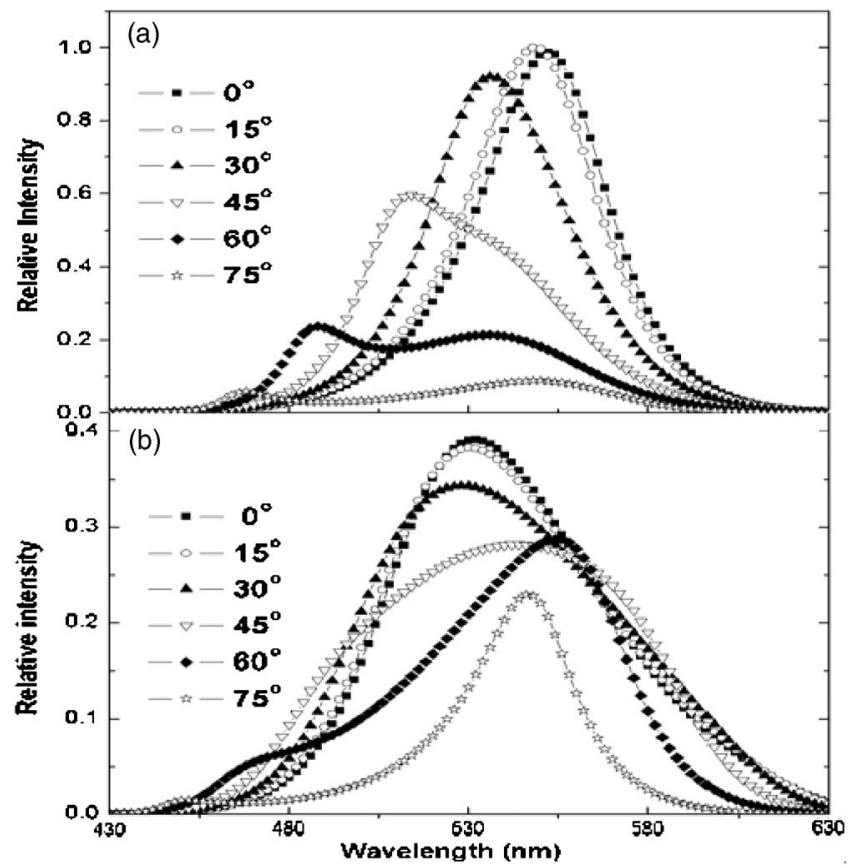

FIG. 4. EL spectra of the TOLEDs optimized (a) for maximum integrated outcoupling efficiency and (b) for a wide viewing angle with $\zeta=0.03$ and $\gamma=0.85$ at viewing angles of $0^{\circ}, 15^{\circ}, 30^{\circ}, 45^{\circ}, 60^{\circ}$, and $75^{\circ}$. 
thanks the support of seed funding from the University of Hong Kong.

${ }^{1}$ J. E. Burrows, G. Gu, S. R. Forrest, E. P. Vicenzi, and T. X. Zhou, J. Appl. Phys. 87, 3080 (2000).

${ }^{2}$ L. S. Hung, C. W. Tang, M. G. Mason, P. Raychaudhuri, and J. Madathil, Appl. Phys. Lett. 78, 544 (2001).

${ }^{3}$ H. Riel, S. Karg, T. Beierlein, W. Rieß, and K. Neyts, J. Appl. Phys. 94, 5290 (2003).

${ }^{4}$ R. B. Pode, C. J. Lee, D. G. Moon, and J. I. Han, Appl. Phys. Lett. 84, 4614 (2004).

${ }^{5}$ L. H. Smith, J. A. E. Wasey, and W. L. Barnes, Appl. Phys. Lett. 84, 2986 (2004).

${ }^{6}$ C. C. Wu, C. L. Lin, P. Y. Hsieh, and H. H. Chiang, Appl. Phys. Lett. 84, 3966 (2004).

${ }^{7}$ C. L. Lin, H. W. Lin, and C. C. Wu, Appl. Phys. Lett. 87, 021101 (2005).

${ }^{8}$ H. J. Peng, X. L. Zhu, J. X. Sun, X. M. Yu, M. Wong, and H. S. Kwok, Appl. Phys. Lett. 88, 033509 (2006).

${ }^{9}$ W. Lukosz, Phys. Rev. B 22, 3030 (1980).

${ }^{10}$ G. Bjork, S. Machida, Y. Yamamoto, and K. Igeta, Phys. Rev. A 44, 669 (1991).
${ }^{11}$ E. M. Purcell, Proceedings of the American Physical Society [Phys. Rev. 69, 681 (1946)].

${ }^{12}$ R. H. Jordan, L. J. Rothberg, A. Dodabalapur, and R. E. Slusher, Appl. Phys. Lett. 69, 1997 (1996).

${ }^{13}$ K. Okamoto, I. Niki, A. Shvartser, Y. Narukawa, T. Mukai, and A. Scherer, Nat. Mater. 3, 601 (2004).

${ }^{14}$ N. Takada, T. Tsutsui, and S. Saito, Appl. Phys. Lett. 63, 2032 (1993).

${ }^{15}$ C. L. Lin, T. Y. Cho, C. H. Chang, and C. C. Wu, Appl. Phys. Lett. 88, 081114 (2006).

${ }^{16}$ K. Neyts, J. Opt. Soc. Am. A 15, 962 (1998).

${ }^{17}$ X. W. Chen, W. C. H. Choy, and S. He, IEEE J. Display Technol. 3, 110 (2007).

${ }^{18}$ D. Zwillinger, Handbook of Integration (Jones and Bartlett, Boston, 1992).

${ }^{19}$ R. K. Lee, Y. Xu, and A. Yariv, J. Opt. Soc. Am. B 17, 1438 (2000).

${ }^{20}$ E. D. Palik, Handbook of Optical Constants of Solids (Academic Press, Orlando, 1985)

${ }^{21}$ P. A. Hobson, J. A. E. Wasey, I. Sage, and W. L. Barnes, IEEE J. Sel. Top. Quantum Electron. 8, 378 (2002).

${ }^{22}$ W. C. H. Choy and E. H. Li, IEEE J. Quantum Electron. 33, 382 (1997).

${ }^{23}$ J.-S. Kim, P. K. H. Ho, N. C. Greenham, and R. H. Friend, J. Appl. Phys. 88, 1073 (2000). 